CARDIOVASCULAR MEDICINE

\title{
Long term follow up of rheumatic fever and predictors of severe rheumatic valvar disease in Brazilian children and adolescents
}

\author{
Z M A Meira, E M A Goulart, E A Colosimo, C C C Mota
}

Heart 2005;91:1019-1022. doi: 10.1136/hrt.2004.042762

See end of article for authors' affiliations .....................

Correspondence to: Dr Zilda M Alves Meira, Department of Paediatrics, Universidade Federal de Minas Gerais, Belo Horizonte 31275030 Brazil; zilda.m.a.m@terra. com.br

Accepted 25 October 2004
Objectives: To investigate the progress of rheumatic fever (RF) and the predictors of severe chronic valvar disease.

Design: Patients prospectively followed up since their first attack of acute RF (ARF).

Setting: Universidade Federal de Minas Gerais, Brazil.

Patients: 258 children and adolescents who met the revised Jones criteria for RF. The follow up period ranged from 2-15 years.

Main outcome measures: The presence and severity of mitral or aortic valvar disease were determined by both clinical and Doppler echocardiographic examinations. The variables associated with severe chronic valvar disease were initially identified by the Kaplan-Meier method and, later, by multivariate analysis. Results: Doppler echocardiography of 258 patients studied showed that 186 (72.1\%) developed chronic valvar disease and 41 (15.9\%) progressed to severe chronic mitral or aortic lesions. Of 146 patients who developed carditis, $49(33.6 \%)$ had a normal clinical examination in the chronic phase but only nine (6.2\%) had normal Doppler echocardiographic findings - that is, $40(27.4 \%)$ patients progressed to chronic subclinical valvar disease. Moderate or severe carditis, recurrences of ARF, and mother's low educational level were risk factors in predicting severe chronic valvar diseases.

Conclusion: The increased risk of progressing to severe chronic valvar disease was associated with moderate or severe carditis, recurrences of ARF, and mother's low educational level. Hence, in a country such as Brazil, the options available for disease control are mainly primary and secondary prophylaxis.
1: developing countries such as Brazil, rheumatic fever (RF) is still an important cause of morbidity and mortality among schoolchildren, adolescents, and young adults. Epidemiological studies have shown that, in the absence of acute RF (ARF) recurrences, new lesions may appear or prior lesions may be aggravated, though at lower frequency and severity. The prognosis is directly associated with severity of carditis and recurrent ARF attacks. ${ }^{1-3}$ About $60 \%$ of patients who developed heart failure during the first ARF attack had developed some valvar diseases 10 years later. ${ }^{4}$ However, even these patients have a greater chance of improvement or even regression of the cardiac lesion if they undergo adequate secondary prophylaxis. ${ }^{56}$

Progress in diagnostic methods, particularly the use of Doppler echocardiography, has provided a more precise evaluation of valvar disease. Several studies have shown that the Doppler echocardiogram is an important tool for accurate diagnosis of cardiac involvement. ${ }^{7-11}$ When Doppler examinations are associated with clinical evaluation and laboratory tests, it is possible to determine to what extent RF has affected the heart. ${ }^{7812}$

In the current medical literature, the prognosis of RF has been evaluated in relation to the importance of each variable, such as severity of carditis, prophylactic control of the disease, and socioeconomic conditions. ${ }^{13-15}$ Therefore, the objectives of this investigation were to study the progress of valvar disease by means of clinical and echocardiographic evaluations and to identify the independent variables that predict severe chronic valvar disease.

\section{METHODS}

This prospective study enrolled 258 children and adolescents with diagnosed RF from August 1983 to December 1998, during their first visit to the service. Age at the first ARF attack ranged from 3.2-16.5 years, with a mean (SD) age of 9.0 (2.6) years. All patients were seen at the Division of Cardiology, Department of Paediatrics, Universidade Federal de Minas Gerais, Brazil. It is a reference centre for RF in the metropolitan area of Belo Horizonte, a region inhabited by roughly three million people. Children were followed up on an outpatient basis by the same team of paediatric cardiologists for a period ranging from 2-15 years (mean 5.4 (2.7) years). The committee on clinical investigation approved this study.

The diagnosis of RF was based on revised Jones criteria. ${ }^{16}$ Carditis and chronic rheumatic heart disease were classified according to the criteria adopted by the service. ${ }^{17}$ The acute phase was characterised as the period from the onset of symptoms up to 12 weeks, or before, when normal results for acute phase reactants were found. The diagnoses of established chronic rheumatic disease by clinical examination, ECG, and chest radiography and of chronic rheumatic valvar disease by Doppler echocardiography were considered only two years after the acute phase.

In the acute phase, 109 patients underwent Doppler echocardiography but the results were not included in this study. During the chronic phase, all 258 patients underwent yearly clinical evaluations and Doppler echocardiography, based on clinical indication, but all patients underwent at least one echocardiographic study in the final evaluation. The presence of subclinical chronic valvar disease was assumed after at least two years of the last ARF attack, for the cases with no clinical evidence of heart involvement, but with Doppler echocardiographic indication of valvar thickening with stenosis or regurgitation not defined as physiological leakage. ${ }^{8-10}$ 
During the acute and chronic phases, mitral and aortic valvar lesions were quantified by the Doppler echocardiography criteria adopted by the Echocardiography Laboratory of Universidade Federal de Minas Gerais. ${ }^{17}$ The valvar lesions classified in the Doppler echocardiograms as mild to moderate were determined to have moderate involvement and lesions classified as moderate to severe were analysed as severe. M mode measurements, cross sectional echocardiography, and pulsed, continuous, and colour flow Doppler were performed to assess heart involvement and severity of valvar regurgitation and stenosis. All echocardiographic examinations were carried out by an echocardiographer who was unaware of the patients' cardiac involvement. The echocardiographic devices used were Siemens CF Plus and Hewlett Packard 1500 (Andover, Massachusetts, USA), with 5.5 $\mathrm{MHz}$ and 2.5 MHz transducers.

Since only one patient died during the study, the adverse event (response variable) chosen in the analysis of predictive factors of poor prognosis was occurrence of severe chronic valvar disease. It was defined as the presence of severe mitral or aortic valvar lesions as recorded on the Doppler echocardiogram. The baseline was defined as the date of the onset of the acute phase and the final event was defined as the occurrence of any type of severe mitral or aortic valvar lesion at least two years after the first ARF attack and recorded according to Doppler echocardiographic classification. The variables associated with severe chronic valvar disease were initially identified by the Kaplan-Meier survival estimate. The differences between the classes of explanatory variables were evaluated by the log rank test. Variables with $p<0.25$ were included in the multivariate analysis. The multivariate Cox regression model was used to determine the effect of each intervening variable while controlling for the influence of all other variables on the development of severe chronic valvar diseases. ${ }^{18} 19$

\section{RESULTS}

In the evolutionary analysis of cardiac involvement based on clinical evaluation, we observed that $38(59.4 \%)$ and 11 $(15.7 \%)$ patients had regressing heart disease when carditis was mild and moderate, respectively. No regressions were observed in severe carditis (table 1). Of 258 patients studied, $186(72.1 \%)$ developed chronic valvar disease and 41 (15.9\%) progressed to severe chronic mitral or aortic lesions in Doppler echocardiograms. Seven (6.3\%) of 112 patients with no clinical evidence of carditis developed moderate valvar disease but none developed severe valvar lesion (table 2). One hundred and forty six patients developed carditis and 49 of them $(33.6 \%)$ had a normal clinical examination in the chronic phase but only nine $(6.2 \%)$ had normal Doppler echocardiographic findings-that is, $40(27.4 \%)$ patients had carditis evolving to chronic subclinical valvar disease.

In the univariate analysis (table 3), variables such as race, mother's low educational level, and low family income were

Table 1 Evolution of rheumatic fever in 258 children and adolescents by severity of carditis and severity of chronic heart disease as determined by clinical examination

\begin{tabular}{lrrrrr}
\hline \multirow{2}{*}{$\begin{array}{l}\text { Severity of carditis: } \\
\text { clinical examination } \\
\text { (first attack) }\end{array}$} & \multicolumn{3}{l}{$\begin{array}{l}\text { Severity of chronic rheumatic heart } \\
\text { disease: clinical examination }\end{array}$} \\
\cline { 2 - 5 } & Absent & Mild & Moderate & Severe & Total \\
\hline Absent & 104 & 5 & 3 & 0 & 112 \\
Mild & 38 & 16 & 7 & 3 & 64 \\
Moderate & 11 & 11 & 27 & 21 & 70 \\
Severe & 0 & 3 & 3 & 6 & 12 \\
Total & 153 & 35 & 40 & 30 & 258 \\
\hline
\end{tabular}

Table 2 Evolution of rheumatic fever in 258 children and adolescents by severity of carditis and severity of chronic rheumatic valvar disease

\begin{tabular}{lrrrrr}
\hline \multirow{2}{*}{$\begin{array}{l}\text { Severity of carditis: } \\
\text { clinical examination } \\
\text { (first attack) }\end{array}$} & \multicolumn{3}{l}{$\begin{array}{l}\text { Severity of chronic rheumatic valvar } \\
\text { disease: Doppler echocardiography }\end{array}$} \\
\cline { 2 - 5 } & Absent & Mild & Moderate & Severe & Total \\
\hline Absent & 63 & 42 & 7 & 0 & 112 \\
Mild & 8 & 39 & 12 & 5 & 64 \\
Moderate & 1 & 12 & 28 & 29 & 70 \\
Severe & 0 & 0 & 5 & 7 & 12 \\
Total & 72 & 93 & 52 & 41 & 258 \\
\hline & & & & & \\
\end{tabular}

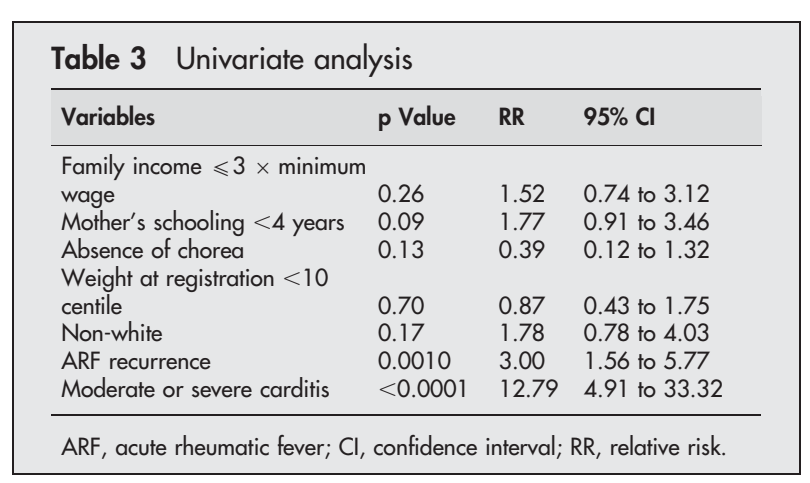

significant risk factors in predicting severe valvar disease. Severe valvar disease was more frequent in non-white patients. The mean schooling of 258 mothers was 4.1 (2.9) years. A high percentage of mothers did not complete elementary school $(86.0 \%)$ and $13.2 \%$ were illiterate. The cut off point for this analysis was four years at school. To assess family income, the cut off point was a salary equivalent to three times the minimum wage in Brazil (US\$240).

ARF recurrence was a risk factor in predicting severe chronic valvar diseases. Of 54 patients who had ARF recurrence, $21(38.8 \%)$ had a poor outcome, whereas only $20(9.8 \%)$ of 204 patients who had no evidence of ARF recurrence progressed to severe chronic rheumatic valvar disease $(\mathrm{p}<0.0001)$. Of 258 patients studied, only one died: a female adolescent who had five recurrent ARF attacks. Regarding nutritional status, the univariate analysis showed that a low z score for weight at enrolment, with a cut off point of -1.29 , was predictive of poor outcome. During the first attack of ARF, $24 \%$ of patients had chorea, alone or associated with arthritis or carditis. Only three (12.5\%) of 24 patients who developed associated carditis progressed to severe valvar disease.

In the multivariate analysis, only three variables were independently associated with severe chronic rheumatic valvar disease: severity of carditis, ARF recurrence, and mother's educational level. The final model also showed a severe interaction between mother's educational level and ARF recurrence (table 4).

The survival curve for chronic rheumatic valvar disease was stratified by severity of carditis: in the high risk group, moderate and severe carditis; and in the low risk group, mild carditis or no carditis. Figure l shows the survival curves for 258 rheumatic patients by the following variables: mother's schooling, severity of carditis, and recurrent ARF attacks.

The interpretation of the mother's educational level variable as a predictive factor of poor outcome took into consideration two situations: absence or presence of ARF recurrence. Among the patients with no ARF recurrences, the 


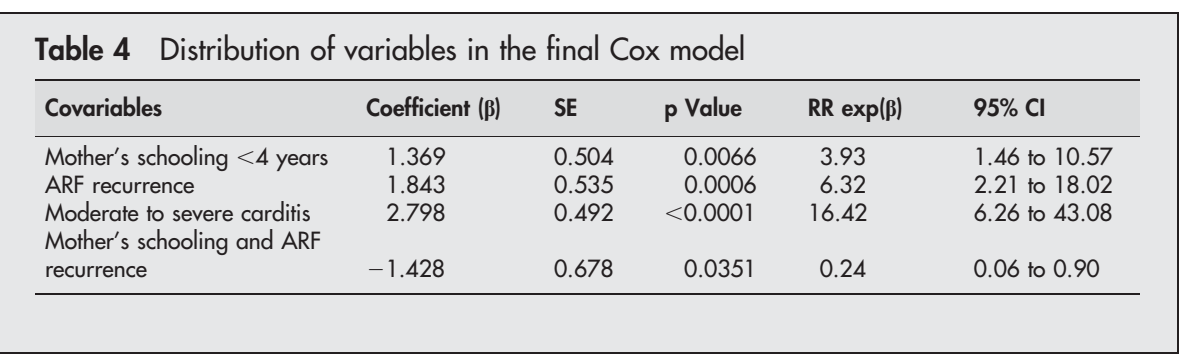

risk of developing severe chronic valvar diseases when their mothers had less than four years of schooling was four times greater than the risk of those whose mothers had studied for four or more years. However, when recurrences were present, the risk of developing chronic valvar diseases was practically the same $(0.94 \cong 1)$ for both groups of children. Among the children whose mothers had studied for four or more years, the risk of acquiring chronic valvar diseases was 6.32-fold greater (95\% CI 2.21 to 18.02) among children with recurrence than among those with no ARF recurrence. Since carditis did not depend on any variable, it could be interpreted by direct observation of the values recorded in the final model. Therefore, the risk of developing severe chronic valvar disease was about 16 times greater for children with moderate or severe carditis than for children with no carditis or for those who developed mild carditis (95\% CI 6.26 to 43.08).

\section{DISCUSSION}

Death is always accepted as an adequate end point in the analysis of predictive factors of poor prognosis. However, since only one patient died in the group followed up in this research, the occurrence of severe chronic rheumatic valvar disease-verified by Doppler echocardiography-was

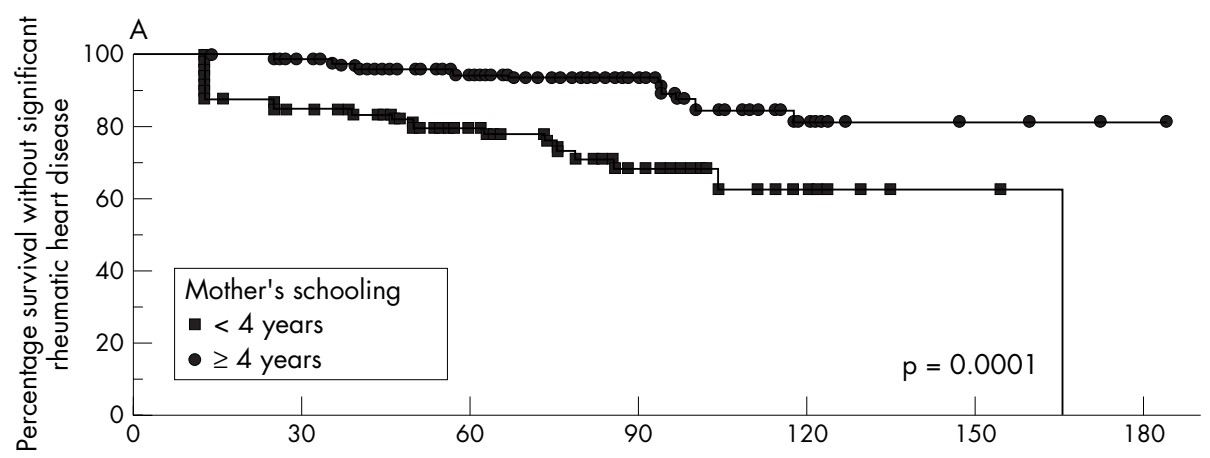

Figure 1 Actuarial survival curves with no severe chronic valvar diseases by (A) mother's educational level, (B) severity of carditis, and (C) recurrent attacks of acute rheumatic fever (ARF).
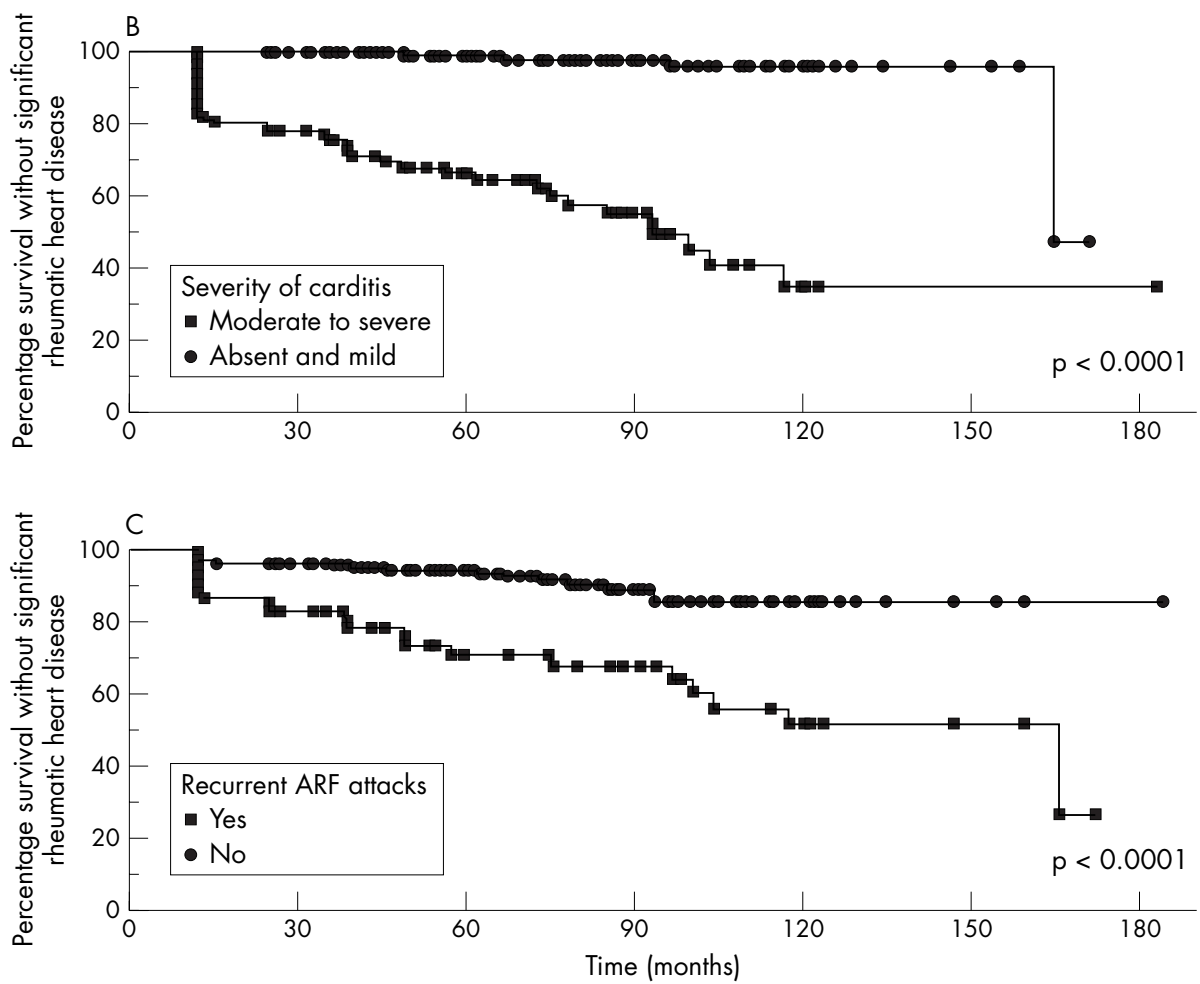
adopted as the end point for this study. To our knowledge, there are no published data on multivariate analysis to assess the RF outcome. Multivariate analysis was applied to the data to control for confounding variables in determining prognosis. Severe chronic rheumatic valvar disease requires at least two years to be defined as such or to develop. ${ }^{1}$ Thus, the determination of the time interval up to the occurrence of this outcome was important for this analysis, resulting in the use of a survival analysis model. ${ }^{19}$

In agreement with data reported by other authors, the poor outcome varied according to severity of carditis. ${ }^{120}$ None of the patients who developed arthritis, either alone or in association with chorea, and who had no evidence of carditis progressed to severe chronic valvar disease. However, of 82 patients with moderate or severe carditis, 69.5\% developed rheumatic heart disease and all patients with severe carditis had clinical evidence of rheumatic heart disease. Chronic subclinical valvar disease was seen in $27.4 \%$ patients, and regression of valvar lesions was less frequently detected by Doppler echocardiography than by clinical assessment.

This investigation detected a high percentage of mothers who did not complete elementary school (86.0\%) and illiterate mothers $(13.2 \%)$. Such results probably reflect the unfavourable socioeconomic conditions of the Brazilian population. Other authors ${ }^{21}$ reported a higher incidence of RF in population segments in which the parents have a low level of schooling. The present study showed that low family income (cut off point of three times the minimum wage) was significant in the univariate analysis as a predictive factor for severe valvar diseases, though it did not remain as a risk factor in the multivariate analysis. Moreover, the z score for weight at enrolment, with a cut off point of 1.29 (corresponding to 10th centile), achieved significance in the univariate analysis but did not remain as an indication of poor outcome in the multivariate analysis. Other studies reported that the evolution of the disease is worse in patients whose nutritional status is below optimum. ${ }^{22}$ The univariate model indicated that race was a significant risk factor in predicting severe valvar disease, with a more frequent poor outcome in non-white patients, but it was not confirmed in the multivariate model. In the medical literature, ethnic and racial differences related to the incidence of this disease are explained by socioeconomic factors, except for the Maori population of New Zealand. ${ }^{23}$ ARF recurrences are known to be directly related to morbidity, mortality, and progression of the disease $e^{2} 2425$ Confirming these data, in our study the number of clinically observed ARF recurrences had a definite influence on progression of the condition.

The multivariate analysis results indicated that there was a significant interaction between mother's educational level and ARF recurrence. In patients who had no ARF recurrence, the risk of developing severe chronic valvar diseases was four times greater for patients whose mothers had studied for less than four years than for those whose mothers had studied for four or more years. In cases of recurrence, the risk of developing severe chronic valvar diseases was the same regardless of their mothers' educational level, showing that ARF recurrence is a very relevant prognostic factor.

Multivariate analysis showed that moderate or severe carditis was an important predictive factor of poor outcome, independent of any other variable. Among the children with moderate or severe carditis, the risk of developing severe chronic valvar diseases was 16.42 times greater than the risk for children who had mild or no carditis. It was shown that severe chronic rheumatic valvar disease was more frequent among patients with moderate or severe carditis, with ARF recurrence, and whose mothers had a low educational level.

As Eisenberg ${ }^{26}$ previously emphasised it is unlikely that poor living conditions, agglomeration, unequal income distribution, low educational level, and malnutrition will change greatly within a short period of time. Hence, in a country such as Brazil, the options available for disease control are mainly primary and secondary prophylaxis.

\section{Authors' affiliations}

Z M A Meira, E M A Goulart, C C C Mota, Department of Paediatrics, Universidade Federal de Minas Gerais, Belo Horizonte, Brazil E A Colosimo, Department of Statistics, Universidade Federal de Minas Gerais

\section{REFERENCES}

1 Décourt LV. Doença reumática. São Paulo: Savier, 1972.

2 Jamal M, Abbas KA. Clinical profile of acute rheumatic fever in children. J Trop Pediatr 1989;35:10-3.

3 Markowitz M, Gordis L. Rheumatic fever. Philadelphia: WB Saunders, 1972.

4 Aziz K, Cheema L, Memon AD. Long-term observations of rheumatic carditis. Cardiol Young 1992;2:254-60.

5 Meira ZMA, Mota CCC, Tonelli E, et al. Evaluation of secondary prophylactic schemes, based on benzathine penicillin $G$, for rheumatic fever in children. J Pediatr 1993; 123:156-8.

6 Tompkins DG, Boxerbaum B, Liebman J. Long-term prognosis of rheumatic fever patients receiving regular intramuscular benzathine penicillin. Circulation 1972;45:543-51

7 Figueroa FE, Fernández MS, Valdés $\mathrm{P}$, et al. Prospective comparison of clinical and echocardiographic diagnosis of rheumatic carditis: long term follow up of patients with subclinical disease. Heart 2001;85:369-70.

8 Abernethy $M$, Bass $N$, Sharpe N, et al. Doppler echocardiography and the early diagnosis of carditis in acute rheumatic fever. Aust $N Z J$ Med 1994;24:530-5.

9 Folger GM Jr, Hajar R. Doppler echocardiographic findings of mitral and aortic valvular regurgitation in children manifesting only rheumatic arthritis. Am J Cardiol 1989;63:1278-80.

10 Minich LL, Tani LY, Pagotto LT, et al. Doppler echocardiography distinguishes between physiologic and pathologic "silent" mitral regurgitation in patients with rheumatic fever. Clin Cardiol 1997;20:924-6.

11 Özkutlu S, Ayabakan C, Saraçlar M. Can subclinical valvitis detected by echocardiography be accepted as evidence of carditis in the diagnosis of acute rheumatic fever? Cardiol Young 2001;11:255-60.

12 Stollerman GH. Current issues in the prevention of rheumatic fever. Minerva Med 2002;93:371-87.

13 Massel BF, Fyler DC, Roy SB. The clinical picture of rheumatic fever: diagnosis, immediate prognosis, course, and therapeutic implications. Am J Cardiol 1958; 1:436-49.

14 Mclaren MJ, Hawkins DM, Koornhof HJ, et al. Epidemiology of rheumatic heart disease in black school children of Soweto, Jonhannesburg. BMJ 1975; iii:474-8

15 Thomas GT. Five-year follow-up on patients with rheumatic fever treated by bed rest, steroids, or salicylate. BMJ 1961;i:1635-9.

16 American Heart Association. Committee on rheumatic fever, endocarditis and Kawasaki disease, council on cardiovascular disease in the young. American Heart Association. Guidelines for the diagnosis of rheumatic fever: Jones criteria, updated 1992, JAMA 1992;268:2069-73.

17 Feigenbaum H. Echocardiography, 4th edn., Philadelphia: Lea \& Febiger 1986.

18 Cox DR, Oakes D. Analysis of survival data. New York: Chapman and Hall, 1984.

19 Healy MJR. Survival analysis. Arch Dis Child 1995;73:374-7.

20 Thevenard RS, Medeiros CCJ. Ecodopplercardiografia na febre reumática. Rev Soc Cardiol Estado de São Paulo 1993;3:43-8.

21 Adanja B, Vlajinac H, Jarebinski M. Socioeconomic factors in the etiology of rheumatic fever. J Hyg Epidemiol Microbiol Immunol 1988;32:329-35.

22 Saraiva LR, Barbalho T, Brindeiro FD. Malnutrition and severe rheumatic heart disease underscoring failure to thrive in an eleven-year-old boy. Cardiol Young 1993;3:79-81.

23 Caughey DE, Douglas R, Wilson W, et al. $\mathrm{HL}-\mathrm{A}$ antigens in Europeans and Maoris with rheumatic fever and rheumatic heart disease. J Rheumatol 1975;2:319-22.

24 Edwards BS, Edwards JE. Congestive heart failure in rheumatic carditis: valvular or myocardial origin? J Am Coll Cardiol 1993;22:830-1.

25 Thakur JS, Negi PC, Ahluwalia SK, et al. Epidemiological survey of rheumatic fever among school children in the Shimla Hills of northern India: prevalence and risk factors. J Epidemiol Community Health 1996;50:62-7.

26 Eisenberg MJ. Rheumatic heart disease in developing world: prevalence, prevention and control. Eur Heart J 1993;152:645-6. 